\title{
Recherches pour une constitution physiocratique
}

Research for a Physiocratic Constitution

\section{Bernard Herencia}

\section{(2) OpenEdition}

\section{Journals}

Édition électronique

URL : https://journals.openedition.org/ahrf/13349

DOI : $10.4000 /$ ahrf. 13349

ISSN : 1952-403X

Éditeur :

Armand Colin, Société des études robespierristes

Édition imprimée

Date de publication : 1 décembre 2014

Pagination : $3-28$

ISBN : 978-2-200-92928-2

ISSN : 0003-4436

\section{Référence électronique}

Bernard Herencia, "Recherches pour une constitution physiocratique », Annales historiques de la Révolution française [En ligne], 378 | octobre-décembre 2014, mis en ligne le 01 décembre 2014, consulté le 01 juillet 2021. URL : http://journals.openedition.org/ahrf/13349 ; DOI : https://doi.org/ 10.4000/ahrf.13349 


\title{
RECHERCHES POUR UNE CONSTITUTION PHYSIOCRATIQUE
}

Bernard HERENCIA

\begin{abstract}
Le physiocrate Paul Pierre Lemercier de la Rivière, parlementaire, ancien administrateur colonial, légiste, publiciste, enrichit dans le contexte de la Révolution les propositions politiques qu'il développe depuis les années 1760 et propose la trame d'une constitution pour le royaume de France. Cet apport méconnu du plus politique des membres de l'école fondée par François Quesnay est notamment assorti de principes et de règles permettant d'exercer un contrôle de constitutionnalité sur l'activité législative des institutions politiques.
\end{abstract}

Mots-clés : Lemercier de la Rivière, physiocratie, constitution, contrôle constitutionnel

Dans ses recherches pour définir le cadre de référence au modèle de despotisme légal qu'il propose et portant le projet d'instaurer un véritable état de droit pour le royaume de France, le physiocrate Paul Pierre Lemercier de la Rivière (1719-1801), parlementaire, ancien administrateur colonial, légiste, publiciste va, dans le contexte de la Révolution, intensifier ses propositions politiques. À partir de la fin des années soixante-dix, après l'échec gouvernemental de Turgot, et avec des tentatives de reprise de ses thèmes par ses successeurs (les assemblées provinciales par exemple), certains physiocrates, dont Lemercier de la Rivière, espèrent revenir aux affaires. Ce contexte s'exacerbe à l'époque des préparatifs des États généraux. Une étude précédente ${ }^{1}$ a montré que Lemercier de la Rivière

(1) Bernard HERENCIA, Physiocratie et gouvernementalité, Thèse ès sciences économiques, Université de Paris Ouest, 2011. 
développe dès les années cinquante les principes de sa réflexion politique. Les publications les plus significatives de ses recherches sont L'Ordre naturel et essentiel des sociétés politiques (1767) - une des œuvres phares de l'école des physiocrates réunie autour de François Quesnay -, les textes des années 1787-1789² et sa dernière œuvre, L'Heureuse nation ou Relations du gouvernement des Féliciens (1792) dans laquelle il présente ses principes dans un texte formellement utopique décrivant la société du peuple imaginaire des Féliciens. La littérature courante réduit généralement l'œuvre politique de Lemercier de la Rivière au concept qu'il a forgé en $1767^{3}$ - le despotisme légal - qui n'est autre chose qu'un régime d'état de droit, puisqu'il s'agit d'assujettir tout le politique et tous les membres de la société, monarque compris, à l'empire de la $\mathrm{loi}^{4}$. Dans les œuvres des années quatre-vingt et quatre-vingt-dix, Lemercier de la Rivière s'efforce de proposer la trame d'une constitution écrite applicable au royaume de France.

Cet article présente les éléments les plus déterminants du dispositif politique que Lemercier de la Rivière finalise dans son projet constitutionnel. Celui-ci conserve les principes de base qu'il a formulés de longue date (sur les pouvoirs par exemple) et les fait coïncider avec des éléments nouveaux (la représentation) pour répondre aux attentes d'une période marquée par les préparatifs des États généraux puis par les premiers travaux de l'Assemblée nationale. Il affirme d'abord la nécessité de doter le royaume d'une constitution écrite puis s'efforce de déterminer une procédure législative propre selon lui à éloigner tout arbitraire du fonctionnement politique du royaume. Dans ce travail il maintient des institutions d'Ancien Régime (la grève parlementaire), retrouve des principes monarchiques tombés en désuétude (les sessions parlementaires régulières et fréquentes), conserve des propositions des premiers temps physiocratiques (les conseils auliques ${ }^{5}$ ), en affine d'autres (la magistrature) et innove (le contrôle constitutionnel). Nous verrons notamment ainsi qu'il produit une constitution d'orientation physiocratique, au travers de propositions constitutionnelles développées,

(2) Paul Pierre Lemercier DE LA RIVIÈRE, Canevas d'un code constitutionnel, Genève, Slatkine, 2011.

(3) Voir Loïc ChARLES et Philippe STEINER, « Entre Montesquieu et Rousseau », dans Etudes Jean-Jacques Rousseau, $\mathrm{n}^{\circ} 11,1999$, p. 83-160.

(4) Pour une étude développée de cette question, voir Bernard HERENCIA, « L'Optimum gouvernemental des physiocrates : despotisme légal ou despotisme légitime ? », Revue de Philosophie Économique, volume $14, \mathrm{n}^{\circ} 2,2013$.

(5) George WeULERSSE, Les Manuscrits économiques de François Quesnay et du marquis de Mirabeau, aux Archives nationales (M. 778 à M. 785), inventaire, extraits et notes, Paris, Geuthner, 1910, p. 27. 
et il espère leur mise en place, dont les fondements tiennent aux conceptions avancées de longue date par les physiocrates : un droit naturel lié à l'économique, une distribution spécifique des pouvoirs ou encore la volonté constante d'établir un contrôle constitutionnel.

\section{La constitution et le processus législatif}

Les textes tardifs de Lemercier de la Rivière ont souvent été interprétés comme une renonciation au despotisme légal et au rétablissement complet du droit des États généraux ${ }^{6}$. Nous verrons que cela est fort éloigné des intentions de l'auteur, même si, de L'Ordre naturel et essentiel des sociétés politiques à L'Heureuse nation, ses conceptions et principes politiques évoluent sensiblement. Mais il s'agit plus d'assouplissements et d'aménagements que de ruptures. Avant de structurer la procédure législative, il affirme la nécessité d'écrire son cadre d'exercice (et propose de rédiger un véritable code constitutionnel) pour le figer. Le terme « constitution », récurrent à l'époque, évoque les principes, modes et organes spécifiques du gouvernement et non simplement l'idée des fondements légaux de l'État. En ce sens elle est, bien plus qu'une base, le résultat, l'émanation du fonctionnement gouvernemental et il cherche à inférer de la base socio-économique de la société et de ses manifestations physiques qui doivent être conformes à l'ordre naturel, la forme institutionnelle supérieure capable d'assurer la pérennité de cette base ${ }^{7}$.

Avant d'aborder la question constitutionnelle, il est nécessaire de fixer la conception physiocratique du droit naturel. C'est essentiellement la question du fondement du droit positif qui a suscité la réflexion sur l'existence d'un droit naturel (« jus naturale ») immuable qui s'opposerait à la variabilité du droit positif en fonction des sociétés et des époques. Platon, dans La République, démontre l'autonomie du droit - fondé sur

(6) Par Chavegrin ou Mergey par exemple. Ernest CHAVEgRIN, « Les Doctrines politiques des physiocrates », dans Raymond CARRÉ DE MALBERG, Mélanges (1933), Paris, Duchemin, 1977, p. 61-70, p. 70 ; Anthony MERGEY, L'État des physiocrates, Aix-en-Provence, PUAM, 2010, p. 323 et 334.

(7) « La constitution est ce qui établit, ce qui détermine la nature et la forme du gouvernement, fixe la condition civile du chef et des membres de la nation; en un mot fait le monarque ce qu'il est, comme monarque, et ses sujets ce qu'ils sont, comme sujets ; forme ainsi ce tout indivisible, ce corps politique auquel est donné le nom de monarchie ». Paul Pierre LEMERCIER DE LA RIVIÈRE, Les Voux d'un François, dans Canevas d'un code constitutionnel, op. cit., p. 108-109. De ce point de vue l'analyse que Lemercier de la Rivière propose de la constitution correspond tout à fait au schéma général d'organisation fondamentale des pouvoirs et de la société tel que l'a dégagé Arnaud Vergne dans son travail de thèse réalisé en 2000 et publié en 2006. Arnaud VERGNE, Les notions de Constitution d'après les cours et assemblées à la fin de l'ancien Régime (1750-1789), Paris, De Boccard, 2006. 
un ordre parfait lié à une nature humaine immuable - par rapport au pouvoir. Les scolastiques, à l'époque médiévale, conservent l'opposition en associant l'homme à la loi divine. Au XVIII' siècle le jusnaturalisme s'impose et Grotius affirme que la raison est normative et qu'elle dicte en l'homme le droit naturel qui est antérieur à tout pouvoir institué. Par la suite, le jusnaturalisme va se structurer à partir d'une distinction fondamentale entre un état de nature (dans lequel les hommes vivent isolément) et un état de société (où les hommes partagent une vie en commun). Dès lors, le droit naturel n'est plus qu'associé à l'individu tandis que la société - considérée comme un artifice découlant d'un hypothétique contrat social - est soumise au droit positif. La question principale qui s'impose alors est celle des rapports entre le droit naturel des individus et le pouvoir de l'État. Pour Hobbes, les hommes, à l'occasion du contrat social, transfèrent leurs droits naturels au souverain tandis que pour Locke, qui met en avant la sociabilité naturelle des hommes, le droit positif est le seul moyen de garantir le droit naturel. Les physiocrates vont développer une approche physique du droit naturel et Quesnay le définit comme « le droit que l'homme a aux choses propres à sa jouissance $»^{8}$ : le droit naturel devient un fondement de la réflexion économique qu'il développe avec ses condisciples. Parmi ceux-ci Lemercier de la Rivière affirme que l'homme est d'emblée un être social et qu'il n'est nul besoin de la parabole d'un quelconque état de nature pour étudier les fondements du droit positif ${ }^{9}$ : celui-ci doit tout entier être dédié à la recherche du produit net le plus élevé - c'est-à-dire l'abondance des denrées permettant la multiplication des hommes - qui est l'intérêt commun que tous les membres de la société, monarque compris, partagent. Ce faisant, le politique dépasse la simple problématique de la paix pour la conservation de l'espèce : abondance et multiplication; ce sont les progrès de la société qui sont invoqués. L'ordre naturel n'est alors pas séparable de la société qu'il organise et dont il impose les structures. Cette immanence de l'ordre naturel réunit les problématiques (la paix, les progrès, etc.) et leurs approches (par le politique, l'économique, etc.). Cet ordre, avec son droit naturel, doit s'imposer despotiquement : c'est ce que Lemercier de la Rivière synthétise avec le concept de despotisme légal ${ }^{10}$.

(8) François QUESNAY, «Le Droit naturel », dans CEuvres économiques complètes et autres textes, Paris, Institut national d'études démographiques, 2005, p.111.

(9) Sur ce point, voir Michael SoNENSCHER, «Physiocracy as a Theodicy », History of Political Thought, $\mathrm{n}^{\circ} 23,2002$, p. 326-39.

(10) Pour un exposé plus complet de la notion physiocratique de droit naturel voir Philippe STEINER, La « Science nouvelle » de l'économie politique, Paris, PUF, 1998, p. 97-101 et BERNARD HERENCIA, Physiocratie et gouvernementalité, thèse citée, p. 291-331. 
Dès 1775, Lemercier de la Rivière ${ }^{11}$ évoque la nécessité d'établir une constitution : « ce n'est pas cependant qu'une société ne puisse confier à un petit nombre, et même à un seul homme, le pouvoir législatif : mais encore faut-il qu'elle ait des loix fondamentales et invariables, auxquelles par conséquent ce Législateur ne puisse absolument déroger $»^{12}$. Cette volonté répond à un programme de recherche ouvert par Quesnay. L'ordre naturel correspond à une constitution naturelle non écrite et la rédiger est évidemment un exercice difficile : sa rédaction doit absolument être fidèle à cette constitution naturelle. Les «maximes » de Quesnay constituent la trame conceptuelle d'une telle constitution et il s'agit de la traduire dans le droit positif. Ce point est une des causes majeures des difficultés de la seconde moitié du XVIII ${ }^{\mathrm{e}}$ siècle, comme le suggère Turgot : « la cause du mal, sire, vient de ce que votre nation n'a point de constitution $\gg^{13}$.

À la fin des années quatre-vingt, et à l'exemple des États-Unis, Lemercier de la Rivière prend acte de la nécessité de rédiger un texte constitutionnel. Il en formule les principes en 1788 (Les Voux d'un François) et rédige un canevas constitutionnel en 1789 (Essais sur les maximes). Il lui a été reproché de ne voir dans la Constitution que la forme de gouvernement et de ne reconnaitre comme «constitution régulière » que la royauté héréditaire et absolue ${ }^{14}$. Il est cependant explicite et entend qu'une constitution devienne « régulière » par « l'établissement de toutes les grandes polices, de toutes les institutions qui doivent nécessairement se réunir pour remplir cet objet [le maintien du droit de propriété, c'est-àdire, de la loi fondamentale entre toute] : voilà le principe d'après lequel vous pouvez vous même la combiner $»^{15}$. Les lois constitutionnelles sont intangibles et le monarque lui-même ne peut les modifier : il ne peut, et ne doit que s'y soumettre. Il faut les recueillir dans un « petit code national » qui « puisse être dans les mains de tout le monde ». Lemercier de la Rivière

(11) Précédemment, il affirme que les « loix fondamentales » sont écrites dans le « code de la nature » et qu'une écriture plus formelle n'est pas utile pour remplacer « l'évidence » et la « raison ». Paul Pierre LEMERCIER DE LA RIVIÈRE, L'Ordre naturel et essentiel des sociétés politiques (1767), Paris, Geuthner, 1910, p 73.

(12) Paul Pierre Lemercier DE LA RIVIÈRE, De l'Instruction publique, Paris, Didot, 1775, 130 p., p. 95. Pour une étude développée sur le rôle de l'instruction dans l'analyse physiocratique, voir Manuela ALBERTONE, Fisiocrati Istruzione e cultura, Turin, Fondazione Luigi Einaudi, 1979.

(13) Anne Robert Jacques TuRgot, Mémoire au roi, sur les municipalités, dans Euvres (1844), 2 vol., Osnabrück, Zeller, 1966, vol. II, p. 504.

(14) Éric GoJosso, « Le Mercier de la Rivière et l'établissement d'une hiérarchie normative », Revue française d'histoire des idées politiques, ${ }^{\circ}$ 20, 2004, p. 285-306, p. 295.

(15) Pierre LEMERCIER DE LA RIVIÈRE, Lettre sur les économistes, dans Canevas d'un code constitutionnel, op. cit., p. 65. Bien plus tard, il évoque un code en forme de déclaration des droits. Id., L'Heureuse nation, 2 t., Paris, Buisson, 1792, t. I, p. 163. 
réclame donc la codification des lois constitutionnelles et attend qu'elles « ne puissent jamais être contrariées par celle de l'administration» car il substitue une dichotomie droit constitutionnel/droit d'administration à l'ancienne distinction droit naturel/droit positif. Il formule alors le principe d'un contrôle de constitutionalité tout à fait inédit, et « cette branche de la constitution monarchique, est ce qui fait la sûreté de cette constitution ${ }^{16}$.

À cette époque, Lemercier de la Rivière tente ainsi de contribuer aux débats institutionnels ouverts avec la crise de l'Ancien Régime et facilités par l'émergence d'une opinion, à nouveau peut-être, favorable aux positions physiocratiques. Cependant, à défaut de parvenir à devenir un véritable acteur des événements en cours, il conçoit, dans son cabinet de travail, une structure constitutionnelle possible pour le politique français des jours à venir, et la publie à plusieurs reprises, guidé probablement par l'espoir d'être rappelé aux affaires. Dans ces années, Lemercier de la Rivière commence à indiquer la structure constitutionnelle, essentiellement législative, qu'il préconise et la lecture des articles des Vøux d'un françois et des Essais sur les maximes permet de dégager la trame générale du processus législatif qu'il propose ${ }^{17}$ (voir le document annexe). Ces diverses dispositions du texte constitutionnel (dont leur auteur minimise la portée en observant qu'il ne s'agit que d'un « canevas ») montrent que l'essentiel du législatif reste dans les mains du monarque et qu'il n'a guère renoncé aux idées de 1767 sur la réunion du législatif et de l'exécutif dans les mêmes mains ${ }^{18}$. Cependant, de nombreuses incohérences ou zones d'ombres persistent, laissant son programme politique inachevé.

Lemercier de la Rivière propose globalement un schéma de la normativité des textes juridiques, avec pour norme fondamentale le droit naturel. Il hiérarchise les règles du droit positif en s'appuyant sur les fonctions politiques. Il dissocie constitution, administration et législation. La première « établit ce qui détermine la nature et la forme du gouvernement, fixe la condition civile du chef et des membres de la nation» pour former « ce tout indivisible, ce corps politique auquel est donné le nom de

(16) Id., Les Voux d'un françois, op. cit., p. 102.

(17) Ce qui ne constitue pas une rupture entre le texte des Vœux d'un François et celui des Essais sur les maximes, comme le soutient Mergey. Anthony MERGEY, L'État des Physiocrates..., op. cit., p. 346.

(18) Cela invalide la thèse selon laquelle avec la publication des Essais sur les maximes (Paul Pierre LEMERCIER DE LA RIVIÈRE, Essais sur les maximes et loix fondamentales, dans Canevas d'un code constitutionnel, op. cit., p. 143-192) « le roi se trouve dépouillé de la puissance législative, dévolue à la Nation représenté par les députés des trois ordres ». Éric GoJosso, «Le Mercier de la Rivière... », op. cit., p. 300-301. 
monarchie $»^{19}$. Il donne là tout d'abord une définition tout à fait moderne de la constitution pour finir par la restreindre au seul régime qu'il souhaite régénérer : la monarchie. La seconde, l'administration, est « la surveillance et la direction de toutes les choses dont la jouissance ou l'utilité sont destinées au corps entier, à tous ses membres sans distinction ». C'est dans le cadre administratif qu'il indique les rôles politiques du souverain et de la Nation : les «choses » sont « des espèces de propriétés communes » puisqu'elles doivent être indépendantes de toute volonté particulière ; ainsi elles relèvent $\mathrm{du}$ 《souverain » comme « représentant de cette volonté commune » toutes les fois où la Nation n'est pas réunie «à son chef ». Lemercier de la Rivière précise enfin la distinction entre la fonction législative, strictement réservée au monarque, et la fonction administrative par laquelle le monarque délègue pour «faire exécuter les volontés du Roy », c'est-à-dire la loi. Il plaide également très tôt ${ }^{20}$ en faveur d'une relative déconcentration en demandant de s'en remettre à l'administration pour exercer l'autorité du roi, toutes les fois où cela est possible, de manière à associer les administrés à la gestion des affaires les concernant.

De la distinction entre administration et constitution naissent deux sortes de lois : les lois variables d'administration et les lois immuables de la constitution (ou lois fondamentales). Les premières sont assujetties à la conjoncture, ce qui les rend changeantes. Lemercier de la Rivière adapte sa conception du droit positif comme simple transcription du droit naturel, quoique «l'administration ne doit être que le développement et l'application des loix de la constitution ». Cela implique une activité législative permanente. Mais ici la puissance législative «ne peut résider que dans le monarque personnellement, puisqu'en cette qualité il est seul et unique administrateur, seul et unique dépositaire de l'autorité suprême instituée pour assurer constamment l'observation des loix ». Cela tempère éminemment le rôle des assemblées qu'il réclame par ailleurs ${ }^{21}$ : le souverain est in fine l'ultime législateur, voire l'unique. Que l'assemblée soit réunie ou non cela ne change rien à l'affaire : le rôle de l'assemblée, bien plus que tempéré, est neutralisé. La nécessité de l'assemblée n'est que théorique, puisque l'intérêt du souverain est indissolublement lié à celui de la Nation ${ }^{22}$. Cependant, l'usage est que «la loi n'oblige que

(19) Paul Pierre LEMERCIER DE LA RIVIÈRE, Les Vœux d'un françois, op. cit., p. 101.

(20) Id., «Exposition sommaire des nouvelles lois », dans Louis-Philippe MAY, Le Mercier de La Rivière, Paris, CNRS, 1978, p. 239-255.

(21) Cf. Bernard HERENCIA, Physiocratie et gouvernementalité, op. cit., p., 394-403.

(22) Id., Paul Pierre LEMERCIER DE LA RIVIÈRE, Les Vøux d'un françois, op. cit., p. 101-102 et Essais sur les maximes, op. cit., Tit. IV, Article II. 
publiée » : les cours de justice souveraines enregistrent les actes du roi, éventuellement après des remontrances préalables voire itératives. Cette procédure associe de fait les parlements au pouvoir législatif à travers l'exercice d'un contre-pouvoir mais les travaux de Lemercier de la Rivière ne prennent que peu en compte cette idée de navette législative. Les secondes - les lois fondamentales - sont les règles juridiques supérieures (et inviolables) de la monarchie : « une loi fondamentale est une loi sans laquelle il ne peut exister une véritable société ; elle est une loi de laquelle doivent dériver toutes les autres lois $»^{23}$. Elles sont aussi nommées « lois du royaume » depuis le $\mathrm{XV}^{\mathrm{e}}$ siècle mais leurs racines sont bien plus anciennes. Intrinsèquement liées à la couronne, elles sont supérieures au roi même : elles protègent les sujets de l'arbitraire et le roi contre lui-même. Lemercier de la Rivière réaffirme ainsi le principe de l'inaliénabilité du domaine royal : les aliénations ne peuvent être qu'issues du «consentement de la nation » et «irrévocables et sans retour ${ }^{24}$. Il mentionne également quelques autres « loix fondamentales et constitutionnelles » : la succession au trône ; l'indivisibilité de la souverainetée ${ }^{25}$; la périodicité des assemblées ; l'indivisibilité de la couronne ; le contrôle de la constitutionalité des lois d'administration et la propriétée ${ }^{26}$. Les lois fondamentales doivent définir et organiser le fonctionnement des institutions ${ }^{27}$.

Lemercier de la Rivière concède sur la forme mais guère sur le fond. Le gouvernement reste monarchique, le roi est unique (Essais sur les maximes, Titre I, Article II) et doit être honoré « comme une divinité tutélaire » (Tit. I, Art. III). Enfin, la monarchie est héréditaire (Tit. VI, Art. VI). Le souverain détient l'intégralité du pouvoir exécutif (Tit. VI, Art. I) et une partie du législatif car il légifère directement en matière de

(23) Id., L'Intérêt commun des Polonois, Archives nationales, Liasse K1317 n 15, p. 28.

(24) Id., Essais sur les maximes, op. cit., Tit. VI, Art. VII.

(25) Deux conceptions de la souveraineté s'opposent dans les débats du début de la Révolution entre une souveraineté populaire issue des analyses de Jean-Jacques Rousseau et l'idée d'une souveraineté nationale (notamment mise en avant par Emmanuel Joseph Sieyès). Dans le second cas, la Nation, peuple sublimé en quelque sorte, est titulaire de la souveraineté et cette version est entérinée par la Déclaration des Droits de l'Homme et du Citoyen du 26 août 1789 (article 3) : «Le principe de toute Souveraineté réside essentiellement dans la Nation ». Nous le verrons, la position de Lemercier de la Rivière peut être rapprochée de cette seconde conception même s'il conserve au monarque la plus grande aptitude à représenter la Nation, tout en introduisant des assemblées représentatives actives et une procédure de contrôle constitutionnel.

(26) Paul Pierre LEMERCIER DE LA RIVIÈRE, Les Vœux d'un françois, op. cit., p. 98-101, 118 et 129 .

(27) Id., L'Heureuse nation, op. cit., t. I, p. 11-12. 
lois d'administration (Tit. VI, Art. II ${ }^{28}$. Il supplée « les lois dans tous les cas sur lesquels il est impossible de statuer », il nomme par exemple aux emplois publics (Tit. I, Art. II) et détient un pouvoir de sanction sur les lois délibérées par la Nation (Tit. IV, Art. III ${ }^{29}$.

La part du pouvoir législatif ne relevant pas du monarque (hormis dans sa prérogative de sanction) est fort restreinte. Deux limites apparaissent, la première est quantitative et la seconde relève du fonctionnement des institutions. Premièrement, les lois « délibérées » et « sanctionnées » par le monarque ne peuvent être abrogées (Tit. IV, Art. III), dès lors, seuls des textes nouveaux (les « autres lois ») visant à "assurer l'exécution de ces mêmes lois [les lois existantes] par les développements et les applications de leurs conséquences $»^{30}$ peuvent être envisagés. Il existe une seconde restriction : les assemblées ordinaires de représentants se tiennent à « époques fixes et périodiques, déterminées par une loi générale »; le terme est ambigu et on peut supposer qu'une loi « générale » n'est qu'une loi relative au fonctionnement de l'administration et donc du ressort du souverain. Quant aux assemblées extraordinaires, leur tenue est décidée par délibération de la Nation (en assemblée ordinaire ?) ou sur convocation du monarque ${ }^{31}$. Ainsi, les réunions des représentants sont la prérogative quasi-exclusive du monarque, puisque les inter-sessions sont plus longues que les sessions elles-mêmes.

La procédure législative que Lemercier de la Rivière propose et que nous venons de schématiser appelle encore quatre précisions. Premièrement, nous l'avons indiqué, il distingue les lois de la constitution et celles de l'administration (Tit. IV, Art. IV). Mais il existe dans les Essais sur les maximes un plus grand nombre de types de textes : aux textes délibérés constitutifs et aux textes d'administrations, s'ajoutent des « autres lois » et des « ordres particuliers du monarque » (Tit. IV, Art. V et Tit. VI, Art. V). Les derniers, contrairement aux premières, sont « affranchis de toute forme » et « faute d'avoir un caractère légal, portent nécessairement celui de l'arbitraire $»^{32}$. Deuxièmement, les lois délibérées et « sanctionnées » par

(28) Les textes d'administration peuvent être assimilés à nos actuels règlements. Mergey, L'État des Physiocrates, op. cit., p. 367. Ce rapprochement peut être observé dans le texte de 1792. Paul Pierre LEMERCIER DE LA RIVIÈRE, L'Heureuse nation, op. cit., t. 1, p. 213.

(29) Le monarque n'est donc pas, comme l'affirme Mergey, « le seul et unique législateur ». Anthony Mergey, L'État des Physiocrates..., op. cit., p. 353. Mais plus loin, il note l'indécision de Lemercier de la Rivière (ibidem, p. 36) et finit par affirmer que le prince « n'est plus titulaire du pouvoir législatif » (ibid., p. 392).

(30) Paul Pierre LEMERCIER DE LA RIVIÈRE, Essais sur les maximes, op. cit., Tit. IV, Art. III.

(31) Ibidem, Tit. VIII, Art. I.

(32) Id., Les Veux d'un françois, op. cit., p. 106-107. 
le roi sont les « lois constitutives » ou « fondamentales », ou « nationales » ou encore, « lois du royaume » ((Tit. II, Art. II, Tit. IV, Art. III et Tit. VI, Art. II) ; ces lois relèvent de « la volonté commune de la nation » et de la sanction du souverain qui leur donne force de loi (Tit. IV, Art. II et III). Elles ne peuvent être abrogées. Le vote de l'impôt ne donne pas lieu à une procédure particulière, mais maintient l'esprit des institutions de l'Ancien Régime, puisqu'il relève obligatoirement des « États généraux » (Tit. X, Art. II). Troisièmement, le monarque intervient pour « donner la sanction qui leur [les lois délibérées] donne force de loi » (Tit. IV, Art. II). Le monarque conserve alors la prérogative du législatif jusqu'à son ultime phase. Enfin, le monarque intervient également comme «puissance législative représentative de la nation» (Tit. V, Art. II) et à ce titre il assure "l'exécution de ces mêmes lois [les lois constitutives] par les développements et les applications de leurs conséquences (Tit. IV, Art. III). Le monarque est alors véritablement législateur et se rapproche de la conception de la réunion des pouvoirs, conçue dans L'Ordre naturel et essentiel des sociétés politiques, mais c'est une réunion sous contrôle du Corps national jouant le rôle d'une contre-force.

Ces précisions ne permettent cependant pas de lever toutes les incertitudes. Le schéma législatif de Lemercier de la Rivière révèle également des imprécisions voire des incohérences. Nous en dénombrons quatre principales : deux sur le fond et deux d'ordre terminologique. La première tient à un point de procédure. Dans les Essais sur les maximes Lemercier de la Rivière n'envisage pas la question de la seconde lecture des textes rejetés ou pour le moins le devenir de textes qui resteraient sans « sanction » royale, sans « promulgation » par le Corps national ou encore invalidés par le contrôle opéré par ce Corps national. Par contre, un an plus tôt (dans les Voeux d'un françois ${ }^{33}$ ), le cas est envisagé. S'il s'agit d'un texte constitutionnel, le rejet est définitif. Dans le cas d'un texte d'administration, l'enregistrement est opéré et le texte fait l'objet d'une « exécution provisoire en attendant qu'une Assemblée nationale puisse faire connoître le vœu général de la Nation ». Cela ne suffit cependant pas à lever l'incertitude. D'une part, rien n'est précisé sur le maintien ou non de la « sanction » royale à l'issue de cette nouvelle délibération. D'autre part, le monarque considéré comme "puissance législative représentative de la nation $»^{34}$ peut-il faire l'économie d'une véritable délibération par les États 
généraux ? Cette hypothèse n'est guère cohérente avec le fonctionnement législatif indiqué par Lemercier de la Rivière et ne doit sans doute pas être retenue. La question de la deuxième lecture trouve d'autres éléments de réponse chez les Féliciens : le prince dispose d'un droit de «veto» mais est « alors tenu de convoquer l'Assemblée Nationale, pour lui déférer les décrets auxquels il s'est opposé, et la mettre en état de se prononcer elle-même sur le sort qu'ils doivent avoir ». Mais l'avancée reste partielle, Lemercier de la Rivière n'indique rien sur l'éventuelle «sanction » que le prince pourrait exercer sur les nouveaux textes puisque la procédure en première lecture en prévoit une ${ }^{35}$. La seconde imprécision est également une question de fond. Le roi donne le caractère exécutif aux lois délibérées mais elles font tout de même l'objet d'une promulgation par le Corps national. La troisième est terminologique : le juriste Lemercier de la Rivière est particulièrement imprécis lorsqu'il examine des «lois », délibérées ou non, alors qu'il ne s'agit que de textes non encore promulgués; une seule fois, il utilise l'expression « lois projetées » (Tit. V, Art. III), sans doute s'agit-il des « avis motivés » portés par les assemblées. La dernière imprécision est à nouveau terminologique. La notion de « lois générales » est loin d'être claire, le vocabulaire n'est pas nécessairement stabilisé. Lorsque Lemercier de la Rivière évoque une " loi générale » pour fixer la convocation préétablie et périodique des assemblées, il est possible d'y voir une « loi constitutionnelle » (les époques sont « fixes » et donc figées) et, dans ce cas, on peut assimiler une loi « générale » à un texte constitutionnel générique.

Ajoutons, pour terminer cet examen de la procédure législative qu'en Félicie le monarque, qui ne détient pas directement le législatif, peut agir par ordonnances ${ }^{36}$. En dépit des imprécisions terminologiques précédentes, la modernite ${ }^{37}$ des conceptions de Lemercier de la Rivière approche notre droit constitutionnel actuel : les « lois fondamentales» (notre Constitution); les « autres lois » (nos lois organiques), les « lois d'administrations » (nos règlements), les «avis motivés » et « lois projetées » (nos propositions et projets de lois). Ces textes du droit positif constituent la matière sur laquelle travaille la magistrature que Lemercier de la Rivière missionne

(35) Id., L'Heureuse nation, op. cit., t. I, p. 202.

(36) Ibidem, p. 213-214.

(37) Sur la question de la modernité de la physiocratie, cf. Manuela ALBERTONE, «Que l'autorité souveraine soit unique », dans Sandrine BAUME et Biancamaria FONTANA, Les usages de la séparation des pouvoirs, Paris, Houdiard, 2007, p. 38-68, passim. 
pour veiller à la conformité de ce droit, sa norme fondamentale : le droit naturel.

\section{La magistrature et le contrôle de constitutionnalité}

À côté du couple législatif/exécutif, Lemercier de la Rivière énonce résolument l'indépendance du judiciaire. Parmi les physiocrates, il est, avec Du Pont de Nemours, un des rares à s'être véritablement préoccupé de donner au pouvoir judiciaire des compétences politiques et constitutionnelles $^{38}$. En fait ces compétences sont déjà en place dans les institutions parlementaires de l'Ancien Régime, mais celles-ci présentent de nombreux dysfonctionnements auxquels il tente de remédier et c'est dans ses réflexions sur la magistrature qu'il énonce les principes essentiels sur lesquels il va fonder le contrôle constitutionnel, et au-delà des remèdes, inventer pour prévenir.

«Le droit de dicter des loix ne peut exister sans le pouvoir physique de les faire observer $»^{39}$, par conséquent dicter les lois positives relève de la seule compétence du souverain qui réunit le législatif et l'exécutif. À côté de ce principe récurrent de sa pensée politique Lemercier de la Rivière a toujours réclamé l'indépendance du judiciaire ${ }^{40}$. Dans L'Heureuse nation, il ne raisonne plus sur trois mais sur quatre pouvoirs (le législatif, le judiciaire, l'administratif et l'exécutif) et réaffirme «l'impossibilité physique et morale » que les membres du corps politique « exercent tous ensemble et en même temps, aucun de ces quatre pouvoirs ». Il manifeste également une volonté de délimiter strictement ces pouvoirs pour éviter leur confusion et leurs contradictions ${ }^{41}$, c'est une manière de se rapprocher de la thèse de la distribution des pouvoirs développée par Montesquieu ${ }^{42}$. Lemercier de la Rivière s'en remet à la magistrature pour assurer la fonction de gardefous à l'égard des fondamentaux physiocratiques : « un corps de citoyens institués pour être [...] dépositaires et gardiens de l'évidence même ; qui en cette qualité sont chargés de veiller sans cesse autour de l'autorité législative $»^{43}$. Cette autorité doit servir de filtre entre le souverain et les hommes « ignorants ou mal intentionnés », d'ultime rempart de l'évidence

(38) Mario EINAUd, The Physiocratic Doctrine of Judicial Control, Cambridge, Harvard University Press, 1938, p. 9.

(39) Paul Pierre LEMERCIER DE LA RIVIÈRE, L'Ordre naturel..., op. cit., p. 102.

(40) Ibidem, p. 84.

(41) Paul Pierre LEMERCIER DE LA RIVIÈRE, L'Heureuse nation, op. cit., t. I, p. 101-102.

(42) Charles Louis de Montesquieu, De l'Esprit des lois (1758), 2 t., Paris, GarnierFlammarion, 1979, II-XI-VI.

(43) Paul Pierre LEMERCIER DE LA RIVIÈRE, L'Ordre naturel..., op. cit., p. 86. 
et de garantie en dernier ressort de la « soumission générale » au droit positif, expression du droit naturel. Le pouvoir judiciaire est nécessairement indépendant du souverain ; dans le cas contraire le monarque «jugeroit enfin seul; et par cette raison s'imposeroit l'obligation rigoureuse de ne jamais se tromper bien reconnue pour être au-dessus des forces de l'humanité ${ }^{44}$. Sur ce point, Mably objecte que si le despote peut être sujet à l'erreur et pas les magistrats, alors pourquoi ne pas placer « la puissance législative dans ce corps infaillible $»^{45}$ ? Mais, lorsque Mably suggère de remplacer le monarque par un collectif de magistrats, il demande finalement ce contre quoi s'élève Lemercier de la Rivière : la réunion du législatif et du judiciaire. Sur l'identification de la phase dans laquelle la magistrature doit effectuer son contrôle, suivons provisoirement (en l'état de la pensée de Lemercier de la Rivière en 1767) Einaudi pour qui le contrôle doit être le plus en amont possible, dès la consultation des magistrats au cours de l'élaboration d'un texte législatif ${ }^{46}$. Einaudi surévalue le rôle du contrôle des magistrats sur le despote physiocratique en l'assimilant à la pratique parlementaire des remontrances de cette époque. La surveillance exercée par la magistrature revient de fait à exercer un droit de retrait qui peut faire office d'un droit d'alerte à l'égard du souverain. Cela peut effectivement être rapproché des réponses parlementaires en forme de « suspensions » ou de " grève ", fréquentes au XVIII ${ }^{\mathrm{e}}$ siècle, qui conduisent simplement les parlementaires à un refus de siéger. Cette possibilité de s'opposer à des lois « injustes », est parfois également rapprochée ${ }^{47}$ de la pratique parlementaire des « droits d'enregistrement » ou « des droits de remontrance »; ce n'est pas de cela dont il s'agit dans le système de Lemercier de la Rivière : de tels droits supposeraient pour le souverain la possibilité de recourir aux lettres de jussion ou aux lits de justice, et conduiraient le souverain à imposer une loi injuste ${ }^{48}$. Lemercier de la Rivière n'envisage donc qu'une procédure tenant de la résistance passive : la loi injuste n'est pas appliquée et cette situation doit simplement alerter le souverain et l'amener à rechercher les meilleures lois positives. Ce point est fondamental car il permet dans cette conception de conserver une stricte séparation du législatif et du

(44) Ibidem, p. 114.

(45) Gabriel Bonnot de MABLY, Doutes proposés aux philosophes économistes (1768), dans Euvres complètes, vol. XI, Lyon, Delamollière, 1792, p. 57 et 63-64.

(46) Mario EINAUd, The Physiocratic Doctrine of Judicial Control, op. cit., p. 53.

(47) Marie-Claire LAVAL-REVIGLIO, «Les Conceptions politiques des physiocrates », Revue française de science politique, $\mathrm{n}^{\circ} 2,1987$, p. 181-213, p. 200. cit., p. 97.

(48) Une loi « monstrueuse ». Paul Pierre LEMERCIER DE LA RIVIÈRE, L'Ordre naturel..., op. 
judiciaire tout en prévoyant une procédure de sauvegarde à l'encontre des lois positives non conformes aux lois naturelles. On ne peut donc y voir un «partage de fait de la puissance législative $»^{49}$, bien au contraire, la procédure indiquée par Lemercier de la Rivière donne toute sa portée au terme de « bouclier » qu'il emploie à l'égard de la magistrature qui est pour lui, nous l'avons indiqué, dépositaire et gardienne de la connaissance de l'ordre naturel et missionnée pour veiller constamment autour de l'autorité législative $^{50}$. L'argumentation serait différente si les magistrats avaient la capacité d'annuler une loi. En effet, édicter une loi ou annuler une loi sont deux opérations permettant d'établir une norme générale. Dans les deux cas, il s'agit d'une action législative. Dans les propositions de Lemercier de la Rivière, les magistrats ne peuvent annuler une loi, ils ne peuvent que s'abstenir d'appuyer leurs décisions sur un texte qu'ils réprouvent : ainsi, ils ne légifèrent pas en faisant le choix de rester «muets $»^{51}$. Ce n'est donc pas le despote lui-même qu'ils infléchissent (pour le remettre sur la voie de l'ordre naturel), ils se contentent d'entraver l'exercice de son arbitraire.

Le partage de l'exécutif n'est guère plus envisageable : « si plusieurs administrateurs aperçoivent de grands avantages personnels dans quelques préjudices faits ou à faire à la nation, je demande qui est-ce qui pourra l'empêcher d'être sacrifiée ? $\gg^{52}$. À cette époque Lemercier de la Rivière admet qu'un partage de l'exécutif peut parfois être efficace mais cela ne peut être que temporaire et ne tient qu'aux qualités morales des responsables et non au système qui a permis un tel gouvernement collégial. Il n'envisage cette situation (transitoire) qu'à la condition qu'un corps de magistrats indépendants puisse contrôler l'exécutif ${ }^{53}$. Cela souligne toute la nécessité pour lui d'assujettir le monarque à la loi et d'instaurer les procédures de contrôle indispensables. Ainsi, l'assurance d'une véritable justice ne peut résider que dans l'indépendance de la magistrature. Le droit positif doit d'abord respecter, dans sa propre préparation et sa formulation, une procédure particulière, et le législateur ne peut être magistrat. Il doit exister un pouvoir pour vérifier la conformité des nouvelles lois avec les anciennes et, sans hésiter, Lemercier de la Rivière le confie à la magistrature chargée d'indiquer le véritable sens des lois pour assurer le lien entre gouvernants

\footnotetext{
p. 200 .

(49) Marie-Claire LAVAL-REVIGLIO, « Les Conceptions politiques des physiocrates », op. cit.,

(50) Paul Pierre LEMERCIER DE LA RIVIÈRE, L'Ordre naturel..., op. cit., p. 114.

(51) Ibidem, p. 90.

(52) Ibid., p. 124.

(53) Ibid., p. 138-139.
} 
et gouvernés ${ }^{54}$. Comme l'observe Einaudi les magistrats doivent donc connaitre les principes de la loi naturelle et les fondements rationnels des lois positives qui doivent être exécutées ${ }^{55}$, là même où Lemercier de la Rivière dissocie la « lettre » et la « raison » de la 1 loi $^{56}$, ce qui lui permet d'examiner les deux fonctions principales des magistrats car, au-delà de ces débats, la magistrature est effectivement investie de deux missions principales $^{57}$ : rendre la justice ; garder les lois positives. « Le dépôt et la garde des loix est en fait une fonction partagée par le souverain éclairé, la nation éclairée et les magistrats éclairés »; la magistrature comme dépositaire et gardienne des lois (naturelles et positives) constitue de fait une contre-force évidente au monarque. Ce faisant la disposition rejoint la volonté chez Montesquieu d'ériger le « dépôt » des lois comme contreforce ${ }^{58}$. Cette dualité est clairement exposée par Du Pont de Nemours dès $1768^{59}$ mais n'est traitée que tardivement par Lemercier de la Rivière. Gojosso $^{60}$ suppose que l'idée formulée par le premier est «implicite » pour le second. En fait, ce dernier laisse, dans les années soixante, la question en suspens et n'y revient que dans les années quatre-vingt. Du Pont de Nemours développe cet aspect et rapporte des propos qu'il attribue à Quesnay :

«les hommes ni leurs gouvernements ne les [les lois] font point et ne peuvent point les faire. Ils les reconnaissent comme conformes à la raison suprême qui gouverne l'univers ; ils les déclarent ; ils les portent au milieu de la société ; ils les présentent à l'obéissance des gens de bien, à la conscience même du méchant. - C'est pour cela qu'on dit PORTEUR de loi, législateur, et recueil des lois PORTÉES, légisLATION et qu'on n'a jamais osé dire FAISEUR de loi, légisFACTEUR, ni légisFACTION $»^{61}$.

(54) Mario EInAudi, The Physiocratic Doctrine of Judicial Control, op. cit., p. 40.

(55) Ibidem, p. 41.

(56) Paul Pierre LEMERCIER DE LA RIVIÈRE, L'Ordre naturel..., op. cit., p. 74.

(57) Ibidem, p. $92-93$ et 110.

(58) Pour un rapprochement plus explicite entre le despotisme légal et le concept de gouvernement modéré de Montesquieu, voir Arnaud SKORNICKI, L'Économiste, la cour et la patrie, Paris, CNRS Éditions, 2011, p. 245-247.

(59) Pierre Samuel Du PONT DE NEMOURS, De l'Origine et des progrès d'une science nouvelle (1768), Paris, Geuthner, 1910, p. 349. Dans le cas contraire, le juge serait un « Ministre d'iniquité », un « barbare », « un furieux ». Paul Pierre LEMERCIER DE LA RIVIÈRE, L'Ordre naturel..., op. cit., p. 97.

(60) Éric GoJosso, «Le Mercier de la Rivière... », op. cit., p. 294. Par ailleurs Mergey introduit une confusion en assimilant le devoir indispensable des juges de connaître la « raison des lois » pour pouvoir les appliquer dans l'examen d'un litige avec « le contrôle a priori exercé avant l'enregistrement ». Anthony Mergey, L'État des Physiocrates..., op. cit., p. 173. Sur ce point, cf. Paul Pierre LEMERCIER DE LA RIVIÈRE, L'Ordre naturel..., op. cit., p. 98-99.

(61) Pierre Samuel Du PONT DE Nemours, Maximes du Docteur Quesnay, dans Eugène DAIRE, Physiocrates (1843), Osnabrück, Zeller, 1966, p. 389-390. Du Pont revient sur cette distinction 
Lemercier de la Rivière est bien plus prudent :

« le terme de faire des loix est une façon de parler fort impropre, [...] on ne doit point entendre par cette expression, le droit et le pouvoir d'imaginer, d'inventer et d'instituer des loix positives qui ne soit pas déjà faites, c'est-àdire, qui ne soit pas des conséquences nécessaires de celles qui constituent l'ordre naturel et essentiel de la société $»^{62}$.

Quesnay, à la même époque, expose la même idée : « les lois positives justes ne sont donc que des déductions exactes ou de simples commentaires de ces loix primitives [les lois naturelles] qui peuvent seules suffire aux hommes justes et éclairés ». Dire que toute loi doit être conforme à l'ordre naturel et dans le même temps que tout l'ordre est contenu et émane du droit de propriété, c'est poser ce dernier comme critère ultime ${ }^{63}$ pour jauger la recevabilité d'une loi : une loi est bonne ou mauvaise selon qu'elle respecte ou porte atteinte au droit de propriété. Dans son analyse des textes de Lemercier de la Rivière et de Du Pont de Nemours, le contemporain Béardé de l'Abbaye ne s'y trompe pas : seul le second remet aux magistrats la fonction de «juger» les lois ${ }^{64}$. L'argumentation de Lemercier de la Rivière est la suivante. La nécessité des lois positives implique l'existence de magistrats possédant la science de l'évidence et se devant d' « éclairer » la Nation et, par suite, le souverain porteur de la souveraineté de cette Nation. Il n'en indique pas plus sur la question d'un éventuel contrôle de la magistrature sur le monarque (dont l'idée n'est que suggérée à l'époque de L'Ordre naturel et essentiel des sociétés politiques). La démonstration repose sur une distinction initiale : d'une part la « lettre» de la loi comme disposition textuelle et positive de la loi et, d'autre part, la « raison» de la loi comme motif à l'origine de sa rédaction ; la première est du ressort

en 1789 (Pierre Samuel Du PONT DE NEMOURS, Examen du gouvernement d'Angleterre, Paris, Froullé, 1789, p. 178) et encore en 1805 (Pierre Samuel Du PONT DE NEMOURS, Nécrologue de M. Quesnay de Saint-Germain, dans François QUESNAY, Euvres économiques et philosophiques, Paris, Peelman, 1888, p. 803). Dès 1888, Oncken (rejoignant Schelle) conteste l'authenticité des propos que Du Pont de Nemours fait prononcer à Quesnay dans ce texte et relève d'importantes incohérences terminologiques. Auguste ONCKEN, « Note », dans François QUESNAY, Euvres économiques et philosophiques, op. cit., p. 805 ; Gustave SCHELle, Du Pont de Nemours et l'École physiocratique, Paris, Guillaumin, 1888, p. 374-375. Par ailleurs, contrairement à ce qu'affirme Anthony Mergey, « légisfacteur » n'apparait pas dans L'Ordre naturel et essentiel des sociétés politiques. Anthony MERGEY, L'État des Physiocrates..., op. cit., p. 103 et 358.

(62) Paul Pierre LEMERCIER DE LA RIVIÈRE, L'Ordre naturel..., op. cit., p. 105.

(63) Sur ce point, cf. Marc LAHMER, La Doctrine physiocratique du contrôle juridictionnel, dans Dominique Chagnollaud, (dir.), Aux origines du contrôle de constitutionnalité, Paris, LGDJ, 2003, p. 31-53, p. 43-44.

(64) BEARDÉ DE L'ABBAYE, Recherches sur les moyens de supprimer les impôts, Amsterdam, Rey, 1770, p. 147-148. 
du législatif c'est-à-dire du souverain, et la seconde des magistrats ${ }^{65}$. Les magistrats s'identifient aux lois naturelles, c'est-à-dire qu'ils se pénètrent de leur nature et que ces lois prennent corps en eux qui en deviennent «la bouche $»^{66}$. Cependant, « le premier, le vrai dépositaire et gardien général des loix, c'est la Nation elle-même à la tête de laquelle est le Souverain »; car le souverain est détenteur de la force physique qui permet d'assurer la sûreté au dépôt des lois naturelles. Les magistrats, dans leurs fonctions de juges, sont «caution» de l'évidence envers le souverain et la Nation. Par l'évidence, ils sont dépositaires des lois positives et également des lois naturelles et, par suite, sont gardiens des lois naturelles constituant « la raison primitive » des lois positives et ils doivent la porter à la connaissance du souverain toutes les fois où son opinion s'est égarée, c'est-à-dire éloignée de l'ordre naturel. Ainsi « la loi juge, et le Magistrat prononce : le jugement sur le fond est l'ouvrage de la loi ; et les procédés qui conduisent au jugement sont l'ouvrage du Magistrat». Le recours auprès du souverain n'est alors possible que sur les questions de procédure et ce recours ne peut conduire qu'à l'annulation d'un jugement et au renvoi d'une affaire devant d'autres magistrats ${ }^{67}$. À la marge, donc, le souverain détient une fonction de cassation en cas de manquement dans le travail judiciaire des magistrats ${ }^{68}$. Cette procédure en cassation n'est cependant pas novatrice, à l'époque le pourvoi en Conseil du roi est un recours possible. Lemercier de la Rivière ne fait donc que reprendre une institution en place. Par contre, face à des lois « évidemment injustes » émises par le souverain, les magistrats ne peuvent que cesser d'être les organes des lois : rendre «muettes » les lois injustes ${ }^{69}$. En définitive, le souverain est l'ultime dépositaire des lois positives mais les magistrats en sont l'ultime caution.

En 1767, Lemercier de la Rivière n'éclaire pas plus cette question du contrôle de la magistrature sur le législatif. Ce n'est qu'en 1789 qu'un « corps national » de magistrats est chargé d'enregistrer et de promulguer les lois nouvelles mais n'exerce un contrôle de conformité à l'ordre que sur une partie d'entre-elles. L'essentiel est cependant intangible jusqu'en 1792, les magistrats sont « des loix vivantes ». Lemercier de la Rivière en

(65) Paul Pierre LEMERCIER DE LA RIVIÈRE, L'Ordre naturel..., op. cit., p. 74.

(66) Lemercier de la Rivière reprend ici l'expression de Montesquieu. Charles Louis de MONTESQUIEU, De l'Esprit des lois, op. cit., II-XI-VI.

(67) Paul Pierre LEMERCIER DE LA RIVIÈRE, L'Ordre naturel..., op. cit., p. 87-111 et 196-197.

(68) Ibidem, p. 196.

(69) Ibid., p. 90 et 97. 
souligne la nécessaire indépendance ${ }^{70}$. Il développe enfin sa pensée sur la magistrature «supérieure » et institue un contrôle constitutionnel.

Une recherche récente ${ }^{71}$ s'intéresse à la volonté de Lemercier de la Rivière d'instituer un contrôle juridictionnel des règles de droit positif. Son auteur, Mergey, identifie, dans ce dispositif, un triple contrôle : de constitutionnalité, d'opportunité et de légalité. Le premier, le contrôle de constitutionnalité, vérifie la cohérence des lois avec la norme juridique supérieure que constituent les lois constitutionnelles. Le second, le contrôle d'opportunité, vérifie l'adaptation des actes d'administration aux circonstances dans lesquelles ils sont intervenus. L'opportunité est liée à deux éléments matériels : l'adéquation de la finalité poursuivie par un acte d'administration avec les circonstances et l'adéquation des moyens aux buts poursuivis. Le contrôle d'opportunité porte donc sur l'adaptation de la décision d'administration aux circonstances tandis que l'évaluation de la pertinence des choix d'objectifs relève du contrôle politique. Ainsi, la vérification en "opportunité » apprécie le niveau de respect de l'intérêt commun dans les actes d'administration et ce rapport d'adaptation peut être différencié du rapport de conformité au droit (i. e. de légalité) mais les deux sont liés puisque la bonne administration appelle à l'insertion de l'opportunité dans le contrôle de légalité. Ce dernier constitue le troisième dispositif de contrôle chargé d'analyser la cohérence des règlements avec la loi. Pour Mergey les trois dispositifs sont exécutés par le même organe de la magistrature, nous verrons qu'en fait il s'agit d'une fonction partagée, en raison de la nature des textes législatifs entre trois entités : les États généraux, le monarque et le Corps national gardien des lois. Par ailleurs Mergey $^{72}$ identifie le contrôle de constitutionnalité et celui d'opportunité dans les travaux de Lemercier de la Rivière de 1788 mais exclusivement un contrôle de légalité dans ceux de 1789. Selon lui, à cette dernière date, la constitutionnalité des lois «nationales » n'est plus vérifiée ; en fait, à ce moment de la réflexion de Lemercier de la Rivière, ce type de textes ne fait pas l'objet d'une vérification par le Corps national parce qu'ils sont préalablement délibérés par les États généraux. Dans l'architecture législative globale que Lemercier de la Rivière ébauche, le contrôle de constitutionnalité et de légalité des textes législatifs « délibérés » par les États généraux est du ressort des États généraux et/ou du monarque. Pour les textes «non délibérés », ce double contrôle relève du monarque et du

(70) Id., L'Heureuse nation, op. cit., t. I, p. 185 et 188.

(71) Anthony MERgey, L'État des Physiocrates..., op. cit.

(72) Ibidem, L'État des Physiocrates..., op. cit., p. 401. 
Corps national gardien des lois, mais dans ce cas un texte non-conforme peut recevoir une exécution provisoire en attendant son examen par les États généraux. Globalement, Lemercier de la Rivière ne prend pas la peine de distinguer loi et règlement parce que, nous l'avons montré, c'est l'ensemble du droit positif qui doit être conforme à la norme supérieure que constitue le droit naturel. Dès lors, tous les textes du corpus législatif doivent être conformes au droit naturel (qui énonce les lois fondamentales) et aux textes positifs ${ }^{73}$ qui ne font qu'en expliciter la lettre. Lorsqu'il envisage, rarement, les règlements à proprement parler Lemercier de la Rivière prévoit explicitement un contrôle de légalité ${ }^{74}$. Contrairement à ce qu'affirme Mergey ${ }^{75}$, en 1789 le contrôle de constitutionnalité reste un fondement de la vérification de la production législative dans les conceptions de Lemercier de la Rivière ; ce qu'il rappelle dans ses Essais sur les maximes (Tit. V, Art. III) et ce contrôle reste simultanément un contrôle de légalité. Notons encore qu'en 1788-89, Lemercier de la Rivière introduit implicitement l'idée d'un contrôle d'opportunité dans le travail du Corps national gardien des lois puisqu'il indique la nécessité de vérifier «l'adoption des moyens » nécessaire à l'accomplissement des « vues » du monarque ou encore à la considération de « l'intérêt général » qui doit présider à l'adoption d'une $\mathrm{loi}^{76}$.

\section{Une ouvre inachevée}

Ce contrôle enrichit la construction politique de Lemercier de la Rivière commencée dans les années soixante. Si le dispositif reste entaché d'imprécisions, il n'en constitue pas moins un apport tout à fait novateur dans l'approche du législatif et par sa volonté de rationaliser le fonctionnement des institutions ${ }^{77}$. La structure législative que nous avons mise en évidence montre que le contrôle de constitutionnalité ne se révèle dans toute sa richesse que par apports et enrichissements successifs dans

(73) «Vérifier une loi, c’est la comparer, la combiner avec les anciennes, et principalement avec celles de la Constitution ». Paul Pierre LEMERCIER DE LA RIVIÈRE, Les Voux d'un françois, op. cit., p. 109-110.

(74) Id., L'Heureuse nation, op. cit., t. I, p. 213.

(75) « En 1789, Le Mercier de la Rivière abandonne toute idée de contrôler la constitutionnalité des lois ». Anthony Mergey, L'État des Physiocrates..., op. cit., p. 403.

(76) Paul Pierre LEMERCIER DE LA RIVIÈRE, Les Voux d'un françois, op. cit., p. 103 et Essais sur les maximes, op. cit., p. VI et Tit. IV, Art. V.

(77) Il est cependant utile de noter que d'autres (Joseph André Brun de la Combe, Isaac René Guy Le Chapelier, Emmanuel Joseph Sieyès ou encore Jérôme Pétion de Villeneuve) s'intéressent au principe du contrôle de constitutionnalité dès 1789 mais les véritables propositions n'émergeront qu'au cours de la préparation de la constitution de l'an III. Sur ces réflexions, voir Marco FIORAVANTI, « Sieyès et le jury constitutionnaire : perspectives historico-juridiques », $A H R F, \mathrm{n}^{\circ} 349,2007$, p. 87-103. 
l'ensemble de son œuvre. Notre synthèse du processus législatif qu'il indique appelle quatre précisions importantes relatives à la validation législative, à la validation royale (sanction), aux textes non-contrôlés et au cas particulier des textes constitutionnels.

Premièrement, Lemercier de la Rivière institue une double validation législative. Un Corps national de « dépositaires et gardiens des lois » composé de hauts magistrats reçoit toutes les « lois » avant leur promulgation mais n'exerce son contrôle de conformité avec les « lois nationales » antérieures que sur les textes non délibérés (Essais sur les maximes, Tit. V, Art. II, III et IV). Il ne s'agit pas ici de respecter le travail des représentants de la Nation (puisque les lois sont « sanctionnées » par le contrôle royal) ni même celui du roi (puisque les textes non délibérés du monarque sont contrôlés par le Corps national). Cela semble indiquer une volonté de limiter la procédure à deux contrôles : États généraux + monarque pour les textes délibérés ; monarque + Corps national pour les textes non délibérés. Dans tous les cas la promulgation est de la compétence du « Corps national » (Tit. V, Art. I) et ne peut intervenir qu' après un éventuel contrôle et après enregistrement (Tit. V, Art. II).

Deuxièmement, Lemercier de la Rivière met en place une validation royale des textes législatifs. L'étape de la « sanction » peut être comprise comme une opération de promulgation et l'on ne voit pas pourquoi le Corps national en prononcerait une seconde (Tit. V, Art. II). Il est encore possible de considérer la «sanction» royale comme un droit d'enregistrement. Si l'hypothèse, dans les propositions de Lemercier de la Rivière, de la sanction royale comme droit d'enregistrement est retenue, il s'agit alors d'un important transfert de compétence par rapport à la situation de l'Ancien Régime où le roi légiférait et les parlements enregistraient. Si par contre cet enregistrement n'est que formel, la promulgation par le Corps national devient plus consistante quoique sans procédure de contrôle. En fait le canevas constitutionnel de Lemercier de la Rivière prévoit bien un enregistrement par le « Corps national» (Tit. V, Art. II). Cependant, il ne parvient pas à donner une indication claire de l'opération constitutionnelle donnant force de loi à un texte. Au contraire, il multiplie les indications contradictoires en invoquant, tantôt l'enregistrement, tantôt la sanction (Tit. IV, Art. II), ou encore la promulgation (Tit. V, Art. I). Nous l'avons vu, il n'est pas parvenu non plus à dégager une distinction nette entre « loi » et « projet de loi », cela lui aurait permis de clarifier ses propositions. Ajoutons à cette question en suspens que Quesnay suggère dès les années cinquante 
le principe de la 4 sanction ${ }^{78}$, acte par lequel, dans la constitution anglaise, le roi approuve un texte législatif proposé par le parlement. En France, dès les premiers travaux de l'Assemblée nationale en 1789, les débats s'animent sur la question de la sanction royale. Elle prendra finalement la forme d'un veto suspensif. Dans ce dispositif, la sanction n'est pas la promulgation. La sanction notifie l'approbation royale du travail parlementaire. Dans le schéma de Lemercier de la Rivière, deux scénarios apparaissent. Dans le premier cas, le roi accorde sa «sanction »; deux lectures sont alors possibles : par la sanction il y a partage de fait du législatif entre le monarque et l'assemblée ; le processus législatif ne peut aboutir que par la sanction royale, le législatif est donc tout entier dans la main du monarque (seul ce dernier point de vue concorde avec l'affirmation constante de Lemercier de la Rivière d'un législatif détenu sans partage par le monarque ; chez les Féliciens encore, le roi conserve son pouvoir de « sanction »). Dans le second cas, le monarque refuse la sanction; il a alors, sans conteste, la maîtrise complète du législatif. Un autre manque dans l'architecture constitutionnelle de Lemercier de la Rivière est à souligner : à aucun moment n'est clairement envisagé le devenir de textes qui resteraient sans « sanction » royale ${ }^{79}$. Il ne mentionne qu'allusivement la sollicitation de la $\ll$ nation $»^{80}$.

Troisièmement, des textes réglementaires échappent à tout contrôle $a$ priori. Les « ordres » du roi ne sont pas contrôlés avant leur application. Ils concernent les « détails d'administrations où il ne s'agira que de l'exécution des lois établies, et des objets qui ne peuvent être ni prévus, ni réglés d'avance par elles [« les formes légales »s'imposant aux autres textes] » (Tit. VI, Art. V). L'imprécision ici ouvre à l'arbitraire ; la seule contre-force invoquée ici est «la sagesse souveraine ». Une autre a été évoquée avec l'affirmation que face à un texte injuste, les magistrats peuvent rester muets.

Quatrièmement et plus fondamentalement : les lois « constitutives » ne peuvent être ni abrogées, ni modifiées (Tit. V, Art. III) ; elles sont «nécessairement immuables » sous peine de « dénaturer la monarchie » (Tit. IV, Art. IV), mais plus loin (Tit. XI, Art. II), Lemercier de la Rivière invoque les « lois du royaume qui ne peuvent être changées qu'avec le consentement de la nation ». Ce dernier point, en faisant abstraction de

(78) François QUESNAY, « Notes », dans Victor Riqueti de MIRABEAU et François QUESNAY, Traité de la monarchie (1757-1759), Paris, L'Harmattan, 1999, p. 24.

(79) Il est donc très difficile de faire une lecture unique de la « sanction » dans les textes de Lemercier de la Rivière. Anthony MERGEY, L'État des Physiocrates..., op. cit., p. 359 et 360.

(80) Paul Pierre LEMERCIER DE LA RIVIÈRE, L'Heureuse nation, op. cit., t. I, p. 206. 
cette remarque, lui permet d'introduire un effet de cliquet législatif : « les lois délibérées par la nation et sanctionnées par son chef ne peuvent être ni abrogées, ni changées par le pouvoir législatif établi pour la représenter $»^{81}$. Le droit constitutionnel devant être conforme à l'ordre naturel (« la nature des choses $»^{82}$ ), dès lors qu'il est établi, devient immuable, à défaut le régime serait dénaturé ${ }^{83}$. Le droit nouveau ne peut servir qu'à approcher mieux la lettre de l'ordre naturel, toujours en conformité avec les lois existantes.

Le canevas constitutionnel de Lemercier de la Rivière n'est donc bien qu'une ébauche de texte constitutionnel. Son principal mérite est d'étudier la voie insuffisamment explorée en 1767 du droit positif. Il s'en dégage cependant une volonté de parachever la réforme politique de l'État en offrant une base de rédaction constitutionnelle de grande ampleur. Ce faisant, il est nécessaire de renoncer définitivement à l'image, dans les apports de Lemercier de la Rivière, d'un despotisme légal associé à une supposée souveraineté absolue du monarque ${ }^{84}$.

Einaudi ${ }^{85}$ considère que le contrôle de constitutionnalité est une question présente dans la pensée de Quesnay dès 1758 lorsqu'il propose d'adjoindre au souverain un conseil pour en prévenir les abus et l'arbitraire : c'est le « conseil aulique », mais Quesnay ne dit rien sur son fonctionnement interne et peu sur les remèdes à ses éventuelles erreurs ${ }^{86}$. En fait, le " flambeau de la raison ${ }^{87}$ doit bien sûr éclairer les législateurs, ce sont les manquements dans l'étude des lois fondamentales qui conduisent à proposer des lois injustes; il est donc « très avantageux » que les magistrats « étendent leurs connaissances sur les lois naturelles » et «n'admettent d'autre vérification que l'évidence ${ }^{88}$. Einaudi s'appuie ensuite sur un texte incertain, nous l'avons noté, quant à son auteur :

(81) Id., Essais sur les maximes, op. cit., Tit. IV, Art. III.

(82) Ibidem, Tit. I, Art. I. Toute loi positive doit ainsi doit donc faire l'objet d'un contrôle de «naturalité » «valant comme garantie politique fondamentale. Marc LAHMER, La Doctrine physiocratique..., op. cit., p. 49.

(83) Paul Pierre LEMERCIER DE LA RIVIÈRE, Essais sur les maximes, op. cit., Tit. IV.

(84) Cette thèse est encore fréquente chez les commentateurs, voir, par exemple Liana VARDI, The Physiocrats and the World of the Enlightenment, Cambridge and New York, Cambridge University Press, 2012, p. 169-170.

(85) Mario EINAUd, The Physiocratic Doctrine of Judicial Control, op. cit., p. 29.

(86) François QUESNAY, « Le Droit naturel », dans CEuvres économiques complètes, op. cit., p. 111.

(87) Mario EINAUd, The Physiocratic Doctrine of Judicial Control, op. cit., p. 30.

(88) François QuESNAY, Despotisme de la Chine, dans Euvres économiques complètes, Paris, INED, 2005, p. 1013. 
« les lois sont irrévocables, elles tiennent à l'essence des hommes et des choses ; elles sont l'expression de la volonté de Dieu ; et plus on y réfléchit, plus on les révère. Les ordonnances sont l'ouvrage des hommes. Elles ont pour objet l'exécution des lois. La soumission provisoire leur est due pour le maintien de l'ordre. Mais il est dans leur nature de demeurer sujettes à l'examen, et d'être révocables quand il devient évident qu'elles ne sont pas d'accord avec les lois ${ }^{89}$.

Pour Einaudi ces mots de Quesnay sont les éléments fondamentaux ${ }^{90}$ de la doctrine du contrôle que développeront les successeurs de Quesnay. Reste qu'Einaudi met en évidence l'apparition ancienne du contrôle constitutionnel dans la pensée physiocratique. Mais c'est à l'œuvre de Lemercier de la Rivière qu'il appartient de lui donner consistance.

Le contrôle de constitutionnalité de Lemercier de la Rivière inaugure les travaux sur le principe de la pyramide des normes par lequel les sources du droit sont ordonnancées suivant une conception en vertu de laquelle une norme inférieure n'est valide qu'en l'absence de contradiction avec une norme qui lui est supérieure. Une institution spécifique est chargée du contrôle et, en cas de non respect de ce principe, une procédure de correction ou d'annulation de la norme invalide est enclenchée. Au sommet de la pyramide, Lemercier de la Rivière place une hypothétique norme fondamentale pour laquelle la possibilité de contrôle est impossible : une fiction juridique indépassable constituée par le droit naturel. Cette perspective de contrôle de conformité dans la hiérarchie des textes est en accord avec la problématique soulevée par Bodin (y compris pour la soumission du monarque aux lois) :

« les contracts et testaments des particuliers, ne peuvent deroger aux ordonnances des magistrats, ny les edicts des magistrats aux coustumes, ny les coustumes aux loix generale d'un prince souverain : aussi les loix des princes souverains ne peuvent alterer, ny changer les loix de Dieu et de nature. [...] Si le Prince est obligé aux loix de nature, et que les loix civiles soyent equitables et raisonnables, il s'ensuit bié que les princes sont aussi tenus aux loix civiles ${ }^{91}$.

(89) Pierre Samuel Du PonT De Nemours, Maximes du Docteur, op. cit., p. 390.

(90) Mario EINAUDI, The Physiocratic Doctrine of Judicial Control, op. cit., p. 32.

(91) Jean Bodin, Les Six livres de la République (1576), Lyon, Tournes, 1579, livre I, chapitre VIII, p. 104-105. 
Les textes tardifs de Lemercier de la Rivière apportent cependant une « innovation capitale $»^{92}$ car ils rendent contraignante la hiérarchie normative des textes légaux. Un tel contrôle est notamment repris par Sieyès en 1795. Le canevas constitutionnel de 1789 préserve l'essentiel du corpus politique physiocratique et il n'y a pas globalement de renoncement au despotisme légal. Au contraire l'architecture constitutionnelle proposée par Lemercier de la Rivière traduit sa persistante volonté d'assujettir le monarque à la loi. En dépit de ses insuffisances son canevas constitutionnel exprime, nous l'avons vu, une étonnante modernité ${ }^{93}$ dans sa détermination à fonder un véritable état de droit.

(92) Éric GoJosso, « Le Mercier de la Rivière et l'établissement d'une hiérarchie normative », op. cit., p. 302.

(93) Sur ce point Albertone souligne « une contribution essentielle à la théorie des garanties constitutionnelles ». Manuela ALBERTONE, «Que l'Autorité souveraine soit unique », op. cit., p. 44. 


\section{ANNEXES}

PROCEDURE LEGISLATIVE

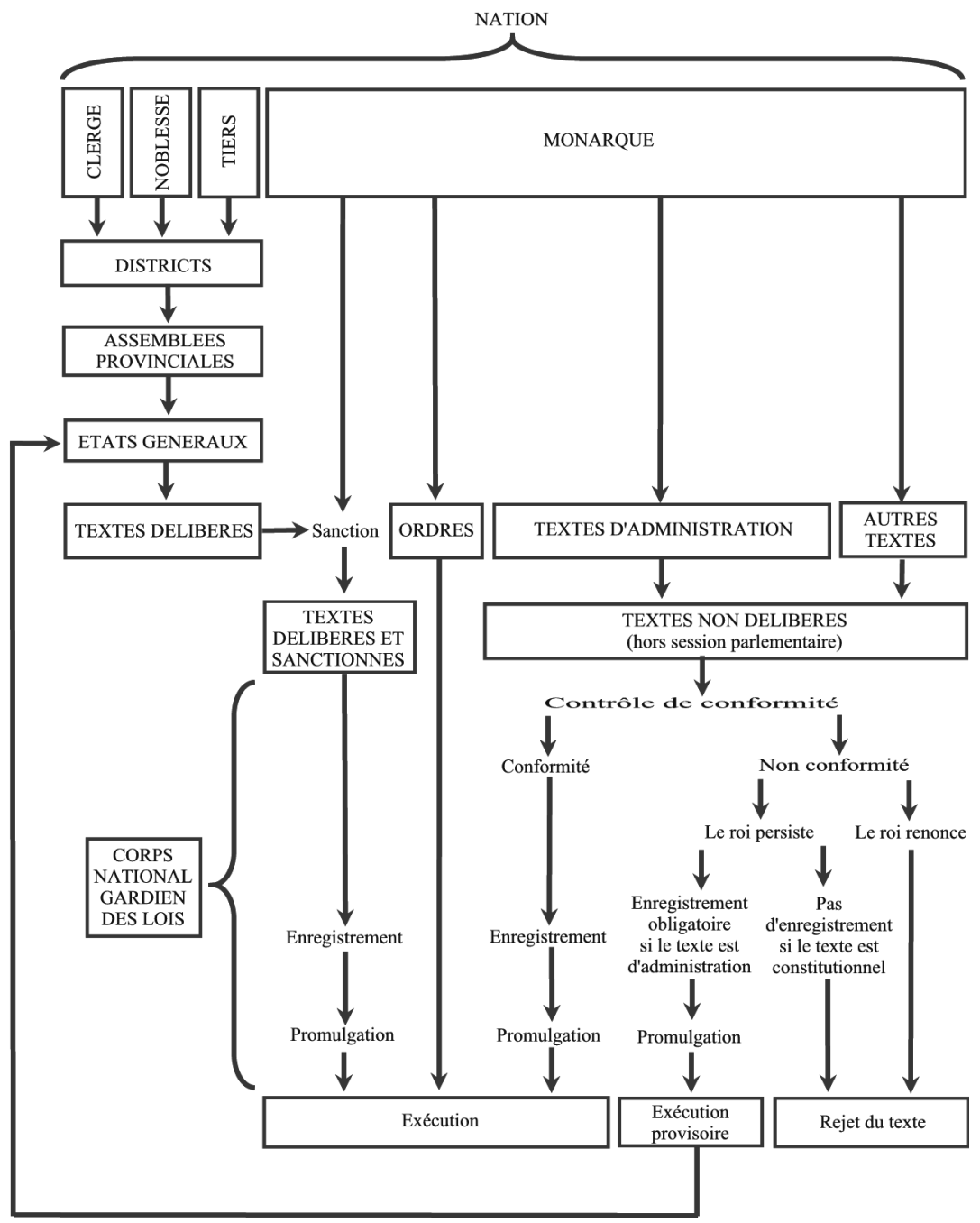


Bernard HERENCIA

Université Paris-Est Marne-la-Vallée, 17 rue Jablinot BP 24, 77101 Meaux Cedex Université Paris-Est, Espaces éthiques et politiques

Institut Hannah Arendt et Largotec.

Université Paul-Valery Montpellier III, C.R.I.S.E.S.

bernard.herencia@u-pem.fr 